\title{
Uma Abordagem para Controle Estatístico do Processo e Gerência Quantitativa de Projetos
}

\author{
Cristina Cerdeiral, Sávio Mendes, Gleison Santos, Mariano Montoni, Rosângela \\ Pinto, Ana Regina Rocha
}

\author{
COPPE/UFRJ - Universidade Federal do Rio de Janeiro \\ Caixa Postal 68511 - CEP 21945-970 - Rio de Janeiro, Brasil \\ \{cerdeiral, savio, gleison, mmontoni, rosangelaps, \\ darocha\} @cos.ufrj.br
}

\begin{abstract}
The statistical process control supports the continuous improvement of the quality and performance of managed processes. Many software organizations aim to improve its processes through this practical, but some fail. One of the causes is the incorrect application of the necessary statistical knowledge in the analysis techniques. This work presents an example of how to statistically control the process and how to quantitatively manage the projects, through the definition of the steps and techniques to be used, providing knowledge for organizations aiming to statistically control its processes regarding the definition and deployment of the analysis techniques and steps.
\end{abstract}

Resumo. O controle estatístico de processos é pré-requisito para apoio à melhoria contínua da qualidade e desempenho de processos. Muitas organizações buscam a melhoria de seus processos através desta prática, mas nem todas alcançam sucesso na sua aplicação. Uma das causas é aplicação incorreta do conhecimento estatístico necessário nas técnicas de análise. Este trabalho apresenta um exemplo de como controlar estatisticamente o processo e gerenciar quantitativamente os projetos, através da definição dos passos e técnicas a serem utilizadas, fornecendo conhecimento para organizações que desejem controlar estatisticamente seus processos na definição e execução dos passos e técnicas de análise.

\section{Introdução}

O controle estatístico de processos é uma metodologia utilizada para avaliar se um dado processo se encontra sob controle estatístico, ou estável estatisticamente, além de auxiliar na melhoria contínua da qualidade e desempenho do processo gerenciado [Florac et al. 1999]. Para um processo estar estável, seu comportamento deve permanecer dentro de certos limites, e, portanto, sua variação deve estar controlada. $\mathrm{O}$ objetivo do controle estatístico é compreender estatisticamente e predizer o comportamento do processo gerenciado, para fins de objetivos de qualidade e desempenho, além de fornecer dados de desempenho, baselines e modelos para a gerência quantitativa de projetos [Chrissis et al. 2003].

O número de organizações em busca da melhoria de seus processos através do controle estatístico tem aumentado [Conradi et al. 2002]. Modelos, como MPS.BR 
(Melhoria de Processo do Software Brasileiro) [MPS.BR 2006] e CMMI (Capability Maturity Model Integration) [Chrissis et al. 2003], ressaltam a importância do controle estatístico dos processos de software nos níveis mais altos de maturidade. Normas, como a ISO/IEC 9001 [2000], ISO/IEC 9004 [2000], ISO/IEC 15504 [2002] e ISO/IEC 15939 [2002] têm dado maior importância à área de medição e análise dos processos em suas versões mais novas. Existem muitos métodos e técnicas para medição e controle estatístico de processos, como PSM (Practical Software Measurement) [McGarry et al. 2002], GQM (Goal Question Metric) [Basili et al. 1994] e Six Sigma [Tayntor 2003]. Apesar disso, nem todas as organizações alcançam sucesso ao aplicarem o controle estatístico aos seus processos [Conradi et al. 2002].

O objetivo da gerência quantitativa de projetos é, a partir dos dados gerados pelo controle estatístico dos processos, gerenciar quantitativamente os processos dos projetos para alcançar os objetivos de qualidade do projeto e de desempenho dos processos [Chrissis et al. 2003]. Relatórios do The Standish Groups's CHAOS [1994, 1997] indicam que $30 \%$ dos projetos de desenvolvimento de software são cancelados antes de serem concluídos e, dos 70\% remanescentes, apenas 30\% atendem aos seus objetivos. Projetos podem fracassar por causa de estimativas imprecisas ou monitoração e controle ineficientes ou ausentes. A gerência quantitativa dos projetos permite que os projetos desviem o mínimo possível do planejado e que as ações corretivas definidas para corrigir desvios em relação ao planejado sejam executadas dentro de um prazo razoável.

Este artigo apresenta uma abordagem para auxiliar o controle estatístico do processo e a gerência quantitativa dos projetos, através da apresentação de indicadores estatísticos e mecanismos para a análise de desempenho dos processos. A abordagem foi desenvolvida com base nas necessidades de uma organização de software localizada na cidade do Rio de Janeiro que pretende executá-la durante os próximos meses. Na próxima seção, são discutidos conceitos do controle estatístico de processos. Os conceitos da gerência quantitativa de projetos são discutidos na seção 3 . A seção 4 apresenta os trabalhos relacionados, a seção 5 apresenta a abordagem proposta e a seção 6, um exemplo de aplicação prática desta abordagem. A seção 7 contém as considerações finais.

\section{Controle Estatístico de Processos}

O controle estatístico de processos é uma técnica para o controle da qualidade e desempenho dos processos, auxiliando na identificação de problemas e nas ações a serem tomadas para que os processos se tornem estáveis estatisticamente [Florac et al. 1999]. Os gráficos de controle foram propostos por Shewhart [1980] para determinar se o processo se encontra estável estatisticamente, ou sob controle. Nestes gráficos, são exibidos os dados coletados de execuções do processo, permitindo a análise do desempenho, estabilidade e capacidade do processo. Os gráficos de controle são construídos através dos cálculos da linha central (representando a média, a tendência central) e dos limites inferior e superior.

Existem diferentes tipos de gráficos de controle, cada um com objetivos e aplicações diferentes. Os gráficos de controle X-bar (gráfico de médias) e seu gráfico de amplitude (R) são utilizados quando a coleta de múltiplos dados sob as mesmas condições e num pequeno período de tempo é possível. Os dados são então, agrupados 
em no mínimo, grupos de tamanho dois, e, através das médias dos grupos e da amplitude dos grupos, o desempenho do processo pode ser analisado. Um exemplo do gráfico X-bar e seu gráfico R de amplitude pode ser visto na Figura 1. Os gráficos de controle XmR (moving range) e seu gráfico de amplitude móvel são utilizados quando o período de tempo entre a coleta dos dados é grande, ou quando existe a necessidade de investigar dado a dado. Os dados são exibidos individualmente, sem grupamentos, e, através da amplitude móvel entre os valores mais próximos no tempo, o desempenho do processo pode ser analisado. Um exemplo do gráfico XmR e seu gráfico de amplitude móvel pode ser visto na Figura 2.
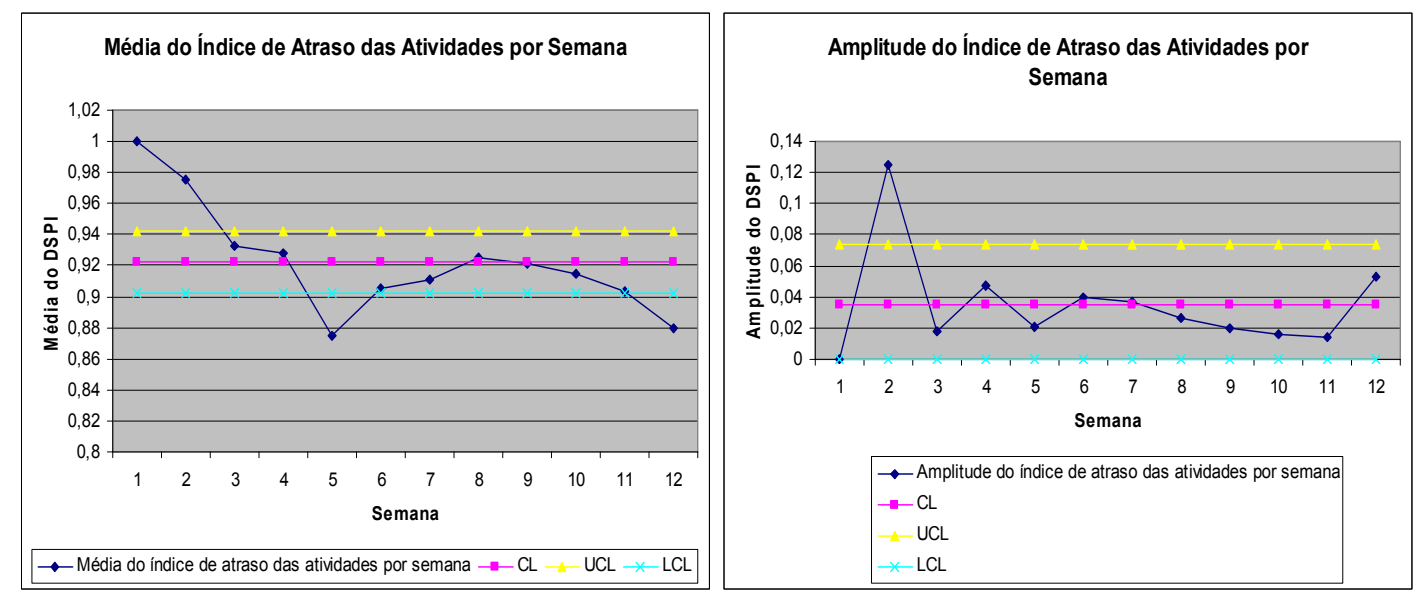

Figura 1 - Gráfico X-bar e seu gráfico de amplitude $\mathbf{R}$
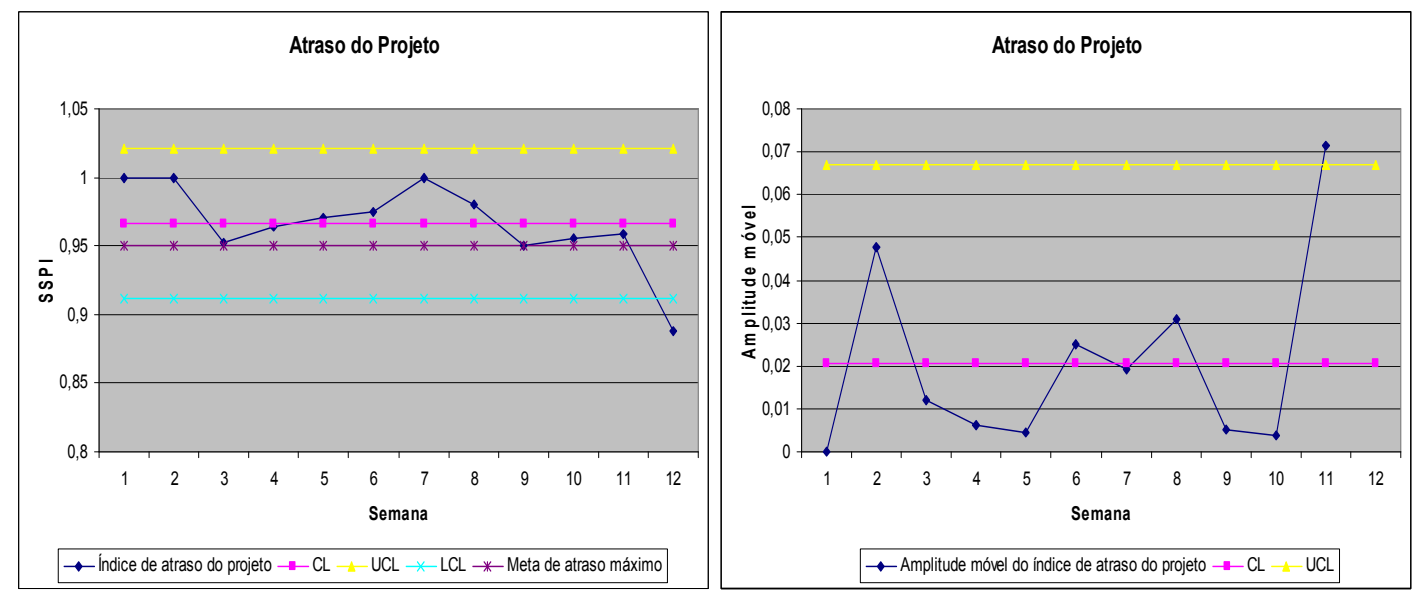

Figura 2 - Gráfico XmR e seu gráfico de amplitude móvel

Os gráficos de controle devem ser utilizados para auxiliar no controle dos processos, permitindo a identificação de quaisquer variações ou desvios. Em [Florac et al. 1999] se encontram quatro testes que podem ser utilizados nos gráficos de controle para detectar algum desvio.

As variações ou desvios podem ser causas comuns ou atribuíveis [Shewhart et al. 1980]. Causas comuns são compostas de variações comuns, que ocorrem o tempo todo em qualquer processo. Causas atribuíveis são compostas de alguma situação ou ação que pode justificar a variação ocorrida, como por exemplo, doença ou demissão de um membro da equipe. A partir da identificação de alguma variação ou desvio, um 
estudo para identificar possíveis causas para tal desvio deve ser realizado. Agrupar os gráficos de controle de forma a facilitar a identificação e confirmação das causas é a abordagem sugerida por Florac et al. [1999]. Uma vez identificadas causas para o desvio, esta é determinada causa atribuível. Caso o desvio tenha impacto negativo, é importante, a partir da identificação de suas causas, realizar um trabalho de prevenção, evitando sua recorrência. Caso o desvio tenha impacto positivo, é importante, a partir da identificação de suas causas, realizar um trabalho de incorporação, provocando sua ocorrência e tornando-a comum.

A análise de desempenho do processo é realizada através dos limites superior e inferior dos gráficos de controle. Se, ao totalizarem 30 pontos no gráfico de controle, nem no gráfico de controle nem no seu respectivo gráfico de amplitude, algum dos quatro testes encontrados em Florac et al [1999] detectarem um desvio, então o processo observado encontra-se estável. Um processo estável, segundo Deming [1986], é um processo com desempenho, custo e qualidade previsíveis e com os efeitos de mudanças mensuráveis. Quando o processo se encontra estável, deve-se gerar uma baseline com os dados dos projetos, que será utilizada para estimar, monitorar e controlar os projetos futuros. Quando o processo não se encontra estável, devem-se estudar os pontos fora dos limites para determinar se são causas atribuíveis ou causas comuns. No caso de serem causas atribuíveis, os gráficos de controle devem ser gerados novamente, isolando o ponto em questão e uma nova análise deve ser realizada. No caso de serem causas comuns, deve-se estudar o processo para diminuir sua variação, com uma maior definição e controle de suas execuções.

A análise de capacidade do processo envolve os objetivos de qualidade e desempenho definidos pela organização. Os gráficos de controle são analisados contra os objetivos desejados e ações corretivas devem ser realizadas sempre que os objetivos não forem alcançados. Deve-se estabilizar os processos e, através dos estudos das causas atribuíveis, diminuir a incidência das mesmas. Com isso, a variação do processo diminui, aproximando os limites superior e inferior da linha central e aumentando cada vez mais a capacidade do processo [Florac et al. 1999].

A baseline de desempenho do processo deve ser elaborada através das informações obtidas de todos os projetos concluídos da empresa que seguiram o mesmo processo padrão de desenvolvimento, contendo as características dos projetos que podem justificar comportamentos diferentes, auxiliando a análise do desempenho, como linguagem de programação utilizada, complexidade do projeto, área de atuação do sistema (base de dados, sistemas de informação, sistemas inteligentes ou especialistas e etc) e fase do processo na qual a informação foi obtida. Quando ocorrem mudanças significativas no processo padrão, uma nova baseline deve ser construída a partir dos projetos concluídos executando o novo processo. Mesmo sem mudanças significativas no processo padrão, mudanças significativas de desempenho podem ocorrer. Um exemplo seria a mudança ocasionada após a organização entrar em uma nova área de atuação.

\section{Gerência Quantitativa de Projetos}

A gerência quantitativa dos projetos envolve estabelecer objetivos de qualidade e desempenho para os projetos, monitorar o projeto e o desempenho do seu processo para 
determinar se os objetivos definidos são alcançados e se o processo é capaz de alcançálos, e tomar ações corretivas sempre que necessário [Chrissis et al. 2003]. As informações de desempenho do processo são geradas pelo controle estatístico do processo, e aqui são utilizadas para gerenciar quantitativamente os projetos.

Como no início de um projeto existem poucas informações para gerenciá-lo quantitativamente, a baseline de desempenho do processo, gerada através do controle estatístico do processo, é extremamente útil. Os gráficos de controle são calculados incluindo as informações dos projetos anteriores juntamente com as já existentes do projeto em andamento. Dessa forma, podem-se identificar desvios no projeto contra a baseline. Com o andamento do projeto, os gráficos de controle podem ser realizados a partir da baseline de processos e a partir dos dados somente do projeto em questão, sendo recomendada a utilização de ambos os tipos de gráficos. Com o auxílio da baseline, verificamos os desvios considerando os vários projetos e, portanto, com uma variância maior, já que cada projeto possui suas particularidades. Os limites tendem a ser mais espaçados da média e estar dentro dos limites é mais fácil. Já com os gráficos de controle a partir somente dos dados do projeto em questão, pode-se verificar se o projeto tem se comportado dentro dos padrões seguidos por ele no passado. Como são dados de um projeto apenas, os limites tendem a ser mais próximos da média, e estar dentro dos limites é mais difícil. Desvios neste tipo de gráfico de controle podem se tornar desvios contra a baseline se não tratados. Logo, a vantagem destes gráficos de controle é a identificação de desvios mais cedo. É importante destacar que os gráficos devem conter cerca de 30 pontos para um nível de confiança aceitável para determinar sua estabilidade.

Através da baseline de desempenho do processo, pode-se estimar o desempenho futuro do processo. A baseline de desempenho do processo pode auxiliar muito nas estimativas de tempo e esforço do projeto, pois existe uma grande probabilidade $(99,9997 \%)$ desses valores não ultrapassarem os limites superiores dos gráficos de controle da baseline. Em caso de desvios contra a baseline de processos, o comportamento dessas variáveis pode ser estimado com maior segurança através dos gráficos de controle construídos a partir somente dos dados do projeto em questão.

\section{Trabalhos Relacionados}

Wang et al. [2006] definiram uma abordagem baseada em estatística para a construção e refinamento de uma baseline de desempenho dos processos, chamada de BSR. Os autores validaram a abordagem através de sua aplicação em algumas organizações.

Komuro [2006] descreve alguns exemplos da aplicação do controle estatístico aos processos de desenvolvimento de software, na Hitachi Software. Características dos processos de desenvolvimento de software, a influência que tiveram sobre os resultados do controle estatístico e as lições aprendidas são discutidas. As medidas, gráficos de controle e as análises realizadas são detalhadas e justificadas.

Sargut et al. [2006] estudaram a aplicação do controle estatístico de processos de desenvolvimento de software em organizações emergentes. Foi realizado um estudo de caso, em uma organização emergente, da utilização do controle estatístico em seus processos, especificamente densidade de defeitos, porcentagem de re-trabalho e 
desempenho de técnicas de inspeção foram os dados gerenciados, e as lições aprendidas, dificuldades e conselhos foram relatados e discutidos pelos autores.

Cangussu et al. [2003] propuseram uma variante ao método tradicional de controle estatístico de processos, que utiliza transformações logarítmicas, para permitir o controle estatístico de processos cujo comportamento é melhor descrito por uma exponencial. Uma avaliação da transformação proposta foi realizada utilizando simulação, e um estudo de caso foi conduzido com um projeto da indústria.

\section{Abordagem Proposta}

O controle estatístico necessita de informações sobre as execuções do processo gerenciado, informações estas que alimentam a base de medidas da organização e possibilitam a construção dos gráficos de controle e posteriores análises estatísticas do desempenho do processo.

A organização que serviu como base para o desenvolvimento da abordagem coordena a oferta de consultoria e soluções em tecnologia de informação de um grupo multinacional italiano, com sede também no Brasil na cidade do Rio de Janeiro. A organização conta com uma equipe de 1400 profissionais que acompanha o cliente, tanto na definição do modelo de TI, quanto no desenvolvimento e implementação de sistemas e serviços.

Com base nas necessidades desta organização, que tem atualmente como objetivo principal aumentar a satisfação dos clientes com a redução do atraso dos projetos, um plano de controle estatístico foi desenvolvido para o processo de monitoração e controle de projetos na organização. O plano desenvolvido orienta a escolha das informações a serem coletadas sobre as execuções do processo, fornecendo algumas questões identificadas como essenciais para controlar estatisticamente o processo. Associados a cada questão, são definidos indicadores, com suas medidas básicas, derivadas e gráficos de controle que podem ser construídos a partir destas, para permitir uma análise estatística do desempenho do processo.

O plano foi desenvolvido com base nas necessidades e cenário encontrados na organização citada acima, a partir de medidas que já são coletados pela organização, e cada empresa que deseje controlar estatisticamente este processo deve adaptar o plano de acordo com os seus objetivos. O plano auxilia as organizações a iniciar o controle estatístico e a entender os seus benefícios, orientando na escolha das informações a serem coletadas e na aplicação das técnicas estatísticas. Com a utilização da abordagem e amadurecimento da organização, novas questões e técnicas podem e devem ser adicionadas, conforme as necessidades identificadas.

As questões identificadas nesta abordagem são:

1 Qual o percentual de atraso das atividades dos processos por projeto?

2 Qual o percentual de atraso das fases dos processos por projeto?

3 Qual o percentual de atraso das atividades críticas dos planos de ação gerados a partir de problemas detectados durante a monitoração dos projetos?

4 Qual a quantidade de planos de ação gerados por projeto? 
5 Qual o percentual de atraso no desenvolvimento de requisitos por fase de projeto?

6 Qual a quantidade de reuniões canceladas por parte do cliente por projeto?

Estas questões necessitam que informações ou medidas básicas sejam coletadas, medidas derivadas sejam calculadas, gráficos de controle sejam construídos e analisados, para que possam então ser respondidas. A abordagem define, para cada questão, indicadores estatísticos e não estatísticos a serem estudados com o objetivo de responder as questões de interesse. Os indicadores estatísticos possuem medidas básicas, medidas derivadas e gráficos de controle associados, que permitem uma análise estatística do desempenho do processo gerenciado. Além dos indicadores estatísticos, a abordagem possui alguns indicadores não estatísticos, com o objetivo de auxiliar na determinação da causa de um determinado problema detectado nos gráficos de controle gerados a partir dos indicadores estatísticos.

Para responder a primeira questão, foram definidos os indicadores estatísticos exibidos na tabela 1, sendo gerados dois gráficos de controle a serem analisados. Ambos os indicadores possibilitam detectar um atraso das atividades do projeto maior que o previsto. Os grupamentos são semanais, o que permite que a monitoração seja semanal.

Tabela 1 - Indicadores Estatísticos da Questão 1

\begin{tabular}{|l|l|}
\hline 1 Qual o percentual de atraso das atividades dos processos por projeto? \\
\hline 1.1 Índice de atraso das atividades dos processos por semana por projeto \\
\hline Medidas Básicas & $\begin{array}{l}\text { SPV (Semanal Planned value - valor planejado por semana): A partir } \\
\text { do cronograma planejado para o projeto, calcula-se o SPV somando o } \\
\text { número de atividades planejadas para serem concluídas até a semana } \\
\text { atual, sendo que cada atividade equivale a uma unidade nesta } \\
\text { contagem, independente da duração da atividade. } \\
\text { SEV (Semanal Earned value - valor realizado por semana): Para cada } \\
\text { atividade concluída durante o projeto, deve-se informar a data de início } \\
\text { e fim da execução. Dessa forma, o SEV é a soma do número de } \\
\text { atividades concluídas até a semana atual. }\end{array}$ \\
\hline Medidas Derivadas & SSPI (Semanal Schedule Performance Index) = SEV / SPV \\
\hline Gráficos de Controle & $\begin{array}{l}\text { Gráfico XmR: Para gerar um ponto no gráfico, deve-se calcular a } \\
\text { amplitude móvel entre os valores de uma semana e a semana seguinte. }\end{array}$ \\
\hline 1.2 Média do índice de atraso das atividades dos processos por semana por projeto \\
\hline Medidas Básicas & $\begin{array}{l}\text { DPV (Daily Planned value - valor planejado por dia): A partir do } \\
\text { cronograma planejado para o projeto, calcula-se o DPV somando o } \\
\text { número de atividades planejadas para serem concluídas até o dia atual, } \\
\text { sendo que cada atividade equivale a uma unidade nesta contagem, } \\
\text { independente da duração da atividade. } \\
\text { DEV (Daily Earned value - valor realizado por dia): Para cada } \\
\text { atividade concluída durante o projeto, deve-se informar a data de início } \\
\text { e fim da execução. Dessa forma, o DEV é a soma do número de } \\
\text { atividades concluídas até o dia atual. }\end{array}$ \\
\hline Medidas Derivadas & DSPI (Daily Schedule Performance Index) = DEV /DPV \\
\hline Gráficos de Controle & $\begin{array}{l}\text { Gráfico X-bar: Para gerar um ponto no gráfico, deve-se somar os } \\
\text { valores dos DSPI de cada dia de uma semana e dividir pelo número de } \\
\text { dias. Em outras palavras, o agrupamento deve ser feito por semana. }\end{array}$ \\
\hline
\end{tabular}

Para responder a segunda questão, foi definido o indicador estatístico exibido na tabela 2, sendo gerado um gráfico de controle a ser analisado. O objetivo deste gráfico 
de controle é permitir uma análise dos atrasos do projeto por fases do processo padrão, o que permite a identificação das fases que mais necessitam de monitoração e controle, além de ações corretivas para a melhoria do desempenho desta fase.

Tabela 2 - Indicadores Estatísticos da Questão 2

\begin{tabular}{|l|l|}
\hline 2 Qual o percentual de atraso das fases dos processos por projeto? \\
\hline 2.1 Percentual de atraso das fases dos processos por projeto \\
\hline Medidas Básicas & $\begin{array}{l}\text { QHPF (Quantidade de Horas Previstas para a Fase): A partir do } \\
\text { cronograma planejado para o projeto, onde, para cada atividade a data } \\
\text { de início e fim devem ser estimadas, calcula-se o QHPF somando as } \\
\text { estimativas das atividades de cada fase. } \\
\text { QHRF (Quantidade de Horas Realizadas na Fase): Para cada atividade } \\
\text { concluída durante o projeto, deve-se informar o número de horas gasto } \\
\text { para sua conclusão. Dessa forma, QHRF é a soma das horas gastas nas } \\
\text { atividades de cada fase. } \\
\text { QHT (Quantidade de Horas Totais): Estimativa da quantidade de horas } \\
\text { totais para a conclusão do projeto. }\end{array}$ \\
\hline Medidas Derivadas & $\begin{array}{l}\text { AF (Atraso da Fase) }=\text { QHRF - QHPF } \\
\text { PAFP (Percentual de Atraso da Fase no Projeto) }=(\text { AF / QHT) } * 100\end{array}$ \\
\hline Gráficos de Controle & $\begin{array}{l}\text { Gráfico XmR: Para gerar um ponto no gráfico, deve-se calcular a } \\
\text { amplitude móvel entre os valores de uma fase e a fase seguinte. }\end{array}$ \\
\hline
\end{tabular}

Para responder a terceira questão, foi definido o indicador estatístico exibido na tabela 3, sendo gerado um gráfico de controle a ser analisado. O objetivo deste gráfíco de controle é permitir uma análise dos atrasos do projeto com relação às atividades dos planos de ação gerados. Quando um plano de ação é gerado, normalmente existe uma ou mais não conformidades associadas a ele a serem resolvidas, e atrasos nessas atividades podem acarretar grandes atrasos no projeto.

Tabela 3 - Indicadores Estatísticos da Questão 3

3 Qual o percentual de atraso das atividades críticas dos planos de ação gerados a partir de problemas detectados durante a monitoração dos projetos?

3.1 Percentual de atraso das atividades críticas dos planos de ação por semana por projeto \begin{tabular}{l|l} 
Medidas Básicas & QAC (Quantidade de Atividades Críticas): Cada atividade inserida em
\end{tabular} um plano de ação é caracterizada como crítica ou não. Semanalmente o gerente do projeto deve coletar as informações do número de atividades críticas existentes nos planos de ação.

QAP (Quantidade de Atividades críticas Pendentes): Cada atividade inserida em um plano de ação é caracterizada como crítica ou não. Semanalmente o gerente do projeto deve coletar as informações do número de atividades críticas existentes nos planos de ação que deveriam ter sido executadas, mas que não foram (pendentes).

Medidas Derivadas PAC (Percentual de Atraso de Atividades) $=$ QAP / QAC

Gráficos de Controle Gráfico XmR: Para gerar um ponto no gráfico, deve-se calcular a amplitude móvel entre os valores de uma semana e a semana seguinte. estatísticos:

Para responder a quarta questão, foram definidos os seguintes indicadores não

- 4.1 Número de planos de ação gerados por projeto (PA): Número de planos de ação gerados até o momento no projeto. Conforme esse número cresce, pode-se 
desconfiar que alguma coisa no processo não se encontre conforme o esperado, o que pode gerar muito retrabalho e um aumento no tempo de conclusão das atividades;

- 4.2 Número de planos de ação gerados por fase por projeto (PAF): Número de planos de ação gerados até o momento por fase do projeto. Conforme esse número cresce, pode-se desconfiar que alguma coisa no processo não esteja conforme o esperado na fase em questão, o que ajuda a focar na fase onde podese ter mais retrabalho e aumento no tempo de conclusão das atividades.

Para responder a quinta questão, foi definido o indicador estatístico exibido na tabela 4, sendo gerado um gráfico de controle a ser analisado. O objetivo deste gráfico de controle é permitir uma análise dos atrasos do projeto com relação às atividades diretamente relacionadas com o desenvolvimento de requisitos. Como se trata de uma atividade que exige grande comunicação com o cliente, atrasos nessas atividades podem ter grande impacto no projeto como um todo, causando dificuldades de agendar reuniões com o cliente para o correto andamento do projeto.

Tabela 4 - Indicadores Estatísticos da Questão 5

\begin{tabular}{|l|l|}
\hline 5 Qual o percentual de atraso no desenvolvimento de requisitos por fase de projeto? \\
\hline 5.1 Percentual de atraso no desenvolvimento de requisitos por fase por projeto. \\
\hline Medidas Básicas & $\begin{array}{l}\text { QTR (Quantidade Total de atividades Realizadas relacionadas } \\
\text { diretamente com o desenvolvimento dos requisitos por fase do } \\
\text { projeto): O gerente do projeto deve coletar esta informação } \\
\text { semanalmente. } \\
\text { QRP (quantidade total de atividades planejadas relacionadas } \\
\text { diretamente com o desenvolvimento dos requisitos por fase do } \\
\text { projeto): O objetivo é determinar quantas destas atividades ainda estão } \\
\text { pendentes para cada fase do projeto. O gerente do projeto deve coletar } \\
\text { esta informação semanalmente. }\end{array}$ \\
\hline Medidas Derivadas & RSPI (Requisites Schedule Performance Index) QRP / QTR \\
\hline Gráficos de Controle & $\begin{array}{l}\text { Gráfico XmR: Para gerar um ponto no gráfico, deve-se calcular a } \\
\text { amplitude móvel entre os valores de uma semana e a semana seguinte. } \\
\text { Serão elaborados gráficos diferentes para fases diferentes. }\end{array}$ \\
\hline
\end{tabular}

Para responder a sexta e última questão, foi definido o seguinte indicador não estatístico:

- 6.1 Quantidade de Reuniões canceladas por parte do cliente ocasionando um impacto negativo no prazo das atividades por fase (QRCC): Número de reuniões agendadas com o cliente a fim de obter mais informações sobre o produto ou serviço a ser desenvolvido que foram desmarcadas, podendo ou não terem sido realizadas no futuro, mas que trouxeram um impacto negativo para o projeto, como por exemplo atraso no cronograma.

Através dos indicadores apresentados, as questões propostas pela abordagem podem ser respondidas, auxiliando a compreensão do desempenho do processo, a verificação de sua estabilidade e de sua capacidade. Com a coleta das informações de execução dos projetos, e a verificação da estabilidade do processo, a baseline do processo pode ser construída. A baseline pode então ser utilizada para gerar estimativas mais confiáveis e gerenciar quantitativamente os projetos. As mesmas questões 
definidas pela abordagem podem ser respondidas para os projetos em andamento, quando gerenciados quantitativamente, permitindo a detecção de desvios de desempenho de seus processos e a tomada de ações corretivas em tempo razoável.

\section{Exemplo de Aplicação da Proposta}

Para auxiliar a compreensão da aplicação da proposta, os exemplos abaixo foram construídos, com dados hipotéticos. No primeiro exemplo, o processo de encontra estável e uma baseline de desempenho do processo é utilizada para estimar o prazo das atividades de um projeto e para monitorar o desempenho do projeto:

"A baseline de desempenho do processo de monitoração e controle de projetos possui, para cada atividade do processo, o tempo e esforço gastos para concluí-la. Com base nesses dados, gráficos de controle de cada atividade em cada projeto podem auxiliar a estimar com maior segurança os valores do novo projeto. Suponha que os dados disponíveis, para a primeira atividade do processo sejam os disponíveis na tabela 5 , contendo 13 projetos onde a atividade foi realizada com o esforço de um homem hora e outros 13 projetos onde a atividade foi realizada com o esforço de dois homens hora.

Tabela 5 - Dados da baseline de desempenho dos projetos para a atividade 1 do processo padrão

\begin{tabular}{|l|l|l|l|l|}
\hline Atividade 1 & \multicolumn{5}{l|}{} \\
\hline Projeto & $\begin{array}{l}\text { Horas } \\
\text { realizadas }\end{array}$ & $\begin{array}{l}\text { Horas totais } \\
\text { do projeto }\end{array}$ & Índice de duração & Esforço (h/h) \\
\hline Projeto 1 & 3 & 950 & 0,003157895 & 1 \\
\hline Projeto 2 & 3,5 & 880 & 0,003977273 & 1 \\
\hline Projeto 3 & 3 & 655 & 0,004580153 & 1 \\
\hline Projeto 4 & 3,5 & 880 & 0,003977273 & 1 \\
\hline Projeto 5 & 4 & 810 & 0,004938272 & 1 \\
\hline Projeto 6 & 4,5 & 900 & 0,005 & 1 \\
\hline Projeto 7 & 3 & 820 & 0,003658537 & 1 \\
\hline Projeto 8 & 3,5 & 709 & 0,00493653 & 1 \\
\hline Projeto 9 & 4 & 850 & 0,004705882 & 1 \\
\hline Projeto 10 & 3,5 & 930 & 0,003763441 & 1 \\
\hline Projeto 11 & 4 & 890 & 0,004494382 & 1 \\
\hline Projeto 12 & 4,5 & 1050 & 0,004285714 & 1 \\
\hline Projeto 13 & 3 & 860 & 0,003488372 & 1 \\
\hline Projeto 1 & 2 & 600 & 0,003333333 & 2 \\
\hline Projeto 2 & 2,5 & 750 & 0,003333333 & 2 \\
\hline Projeto 3 & 2 & 800 & 0,0025 & 2 \\
\hline Projeto 4 & 1,5 & 650 & 0,002307692 & 2 \\
\hline Projeto 5 & 2 & 700 & 0,002857143 & 2 \\
\hline Projeto 6 & 2,5 & 760 & 0,003289474 & 2 \\
\hline Projeto 7 & 1 & 570 & 0,001754386 & 2 \\
\hline Projeto 8 & 2,5 & 770 & 0,003246753 & 2 \\
\hline Projeto 9 & 3 & 850 & 0,003529412 & 2 \\
\hline Projeto 10 & 1,5 & 590 & 0,002542373 & 2 \\
\hline Projeto 11 & 2 & 660 & 0,003030303 & 2 \\
\hline Projeto 12 & 2,5 & 700 & 0,003571429 & 2 \\
\hline Projeto 13 & 1,5 & 500 & 0,003 & 2 \\
\hline
\end{tabular}


Como o tamanho das atividades é dependente do tamanho do projeto, o índice de duração é calculado dividindo um pelo outro. As atividades foram realizadas com esforços diferentes em cada projeto, e as baselines serão divididas pelo esforço adotado. Com base nesses dados, os gráficos XmR podem ser construídos para as atividades com esforço de 1 ou 2 homens/hora, resultando nos gráficos exibidos nas Figuras 3 e 4.
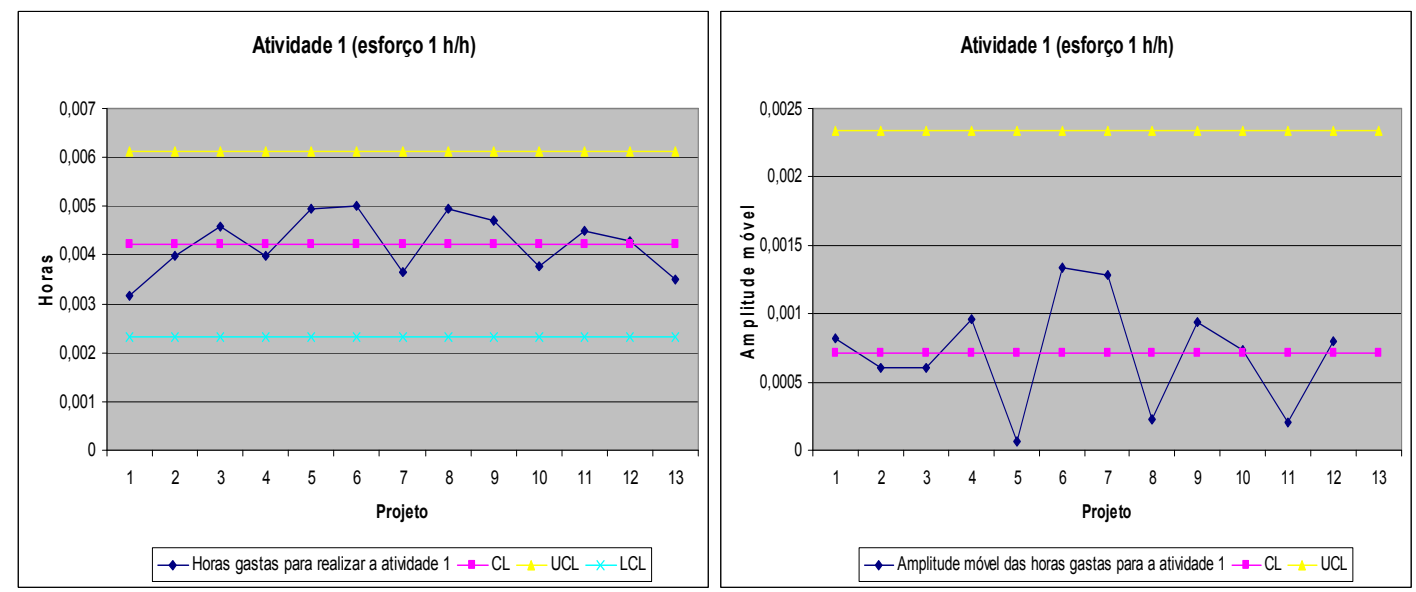

Figura 3 - Gráficos de controle estatístico para a atividade 1 do processo padrão com o esforço de $1 \mathrm{~h} / \mathrm{h}$
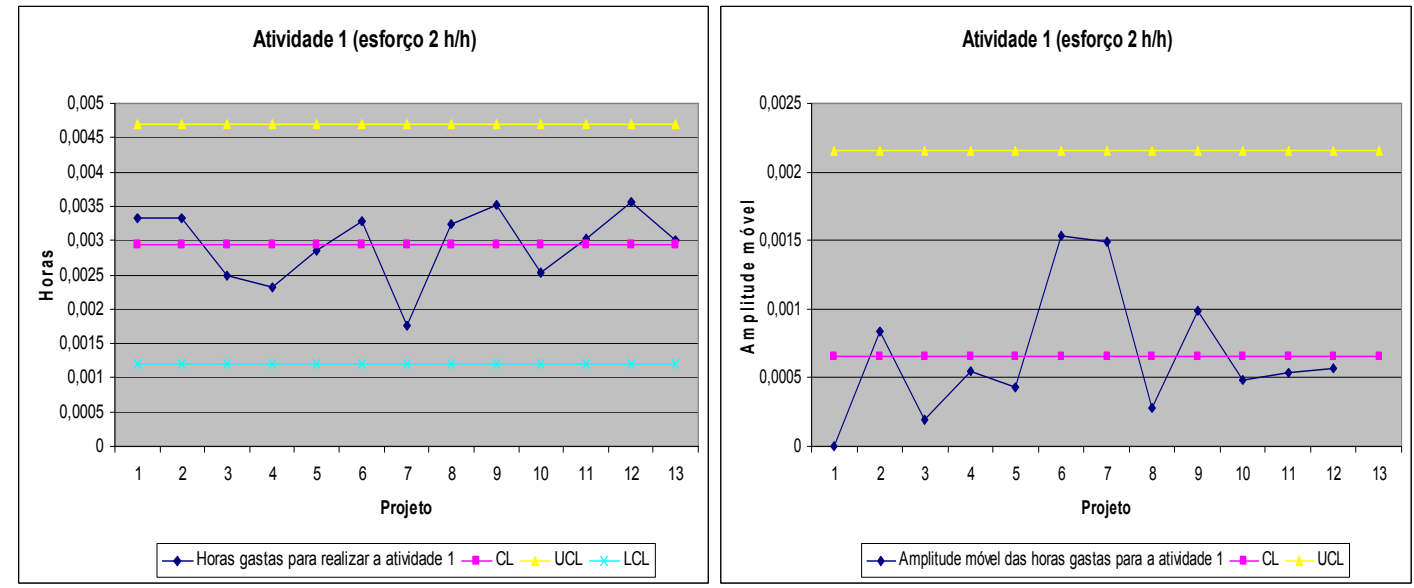

Figura 4 - Gráficos de controle estatístico para a atividade 1 do processo padrão com o esforço de $2 \mathrm{~h} / \mathrm{h}$

Com esses dados da baseline, podemos calcular uma estimativa para o novo projeto. Suponha que seu tamanho seja estimado em 850 horas totais. Multiplicando os valores do gráfico pelo tamanho do novo projeto temos:

- Esforço $1 \mathrm{~h} / \mathrm{h}$ :

Linha central $=3,593782$

Limite superior $=5,209641$

Limite inferior $=1,977922$
- Esforço $2 \mathrm{~h} / \mathrm{h}$ :

Linha central $=2,503945$

Limite superior $=3,993152$

Limite inferior $=1,014738$

Para o caso no qual somente uma pessoa fosse alocada a essa atividade, é relativamente seguro que a atividade 1 do novo projeto não leve mais que cerca de 5,2 
horas e menos que 2,0 horas para ser realizada, sendo mais provável ficar por volta das 3,6 horas. Já para o caso no qual duas pessoas fossem alocadas à essa atividade, é relativamente seguro que a atividade 1 do novo projeto não leve mais que cerca de 4,0 horas e menos que 1,0 hora para ser realizada, sendo mais provável ficar por volta das 2,5 horas. Quanto mais próximo do menor valor for a estimativa, maior o risco assumido pela organização, pois maior a probabilidade de ocorrer atraso na conclusão dessa atividade. Quanto mais próximo do maior valor for a estimativa, menor o risco assumido pela organização, pois menor a probabilidade de ocorrer atraso na conclusão dessa atividade.

Suponha que o gerente do projeto tenha alocado um homem para a atividade $1 \mathrm{e}$ o projeto tenha iniciado suas atividades. Nas semanas iniciais, o gerente deseja se certificar de que o projeto está com o andamento conforme o desempenho esperado, por se tratar de um projeto muito importante para a empresa. Apesar das poucas informações presentes do projeto em questão, o gerente pode utilizar a baseline de processos para gerenciar quantitativamente o projeto. Com a baseline composta das informações de esforço e horas gastas para a atividade 1 do processo para o esforço de um homem/hora (apresentada na Figura 4), o gerente pode verificar se o desempenho do processo do projeto se encontra dentro dos limites desejados, já em sua primeira atividade. Para isso, ele gera o gráfico de controle novamente, agora composto das informações da primeira atividade do projeto e dos projetos anteriores. O gráfico de controle gerado pode ser observado na Figura 5.
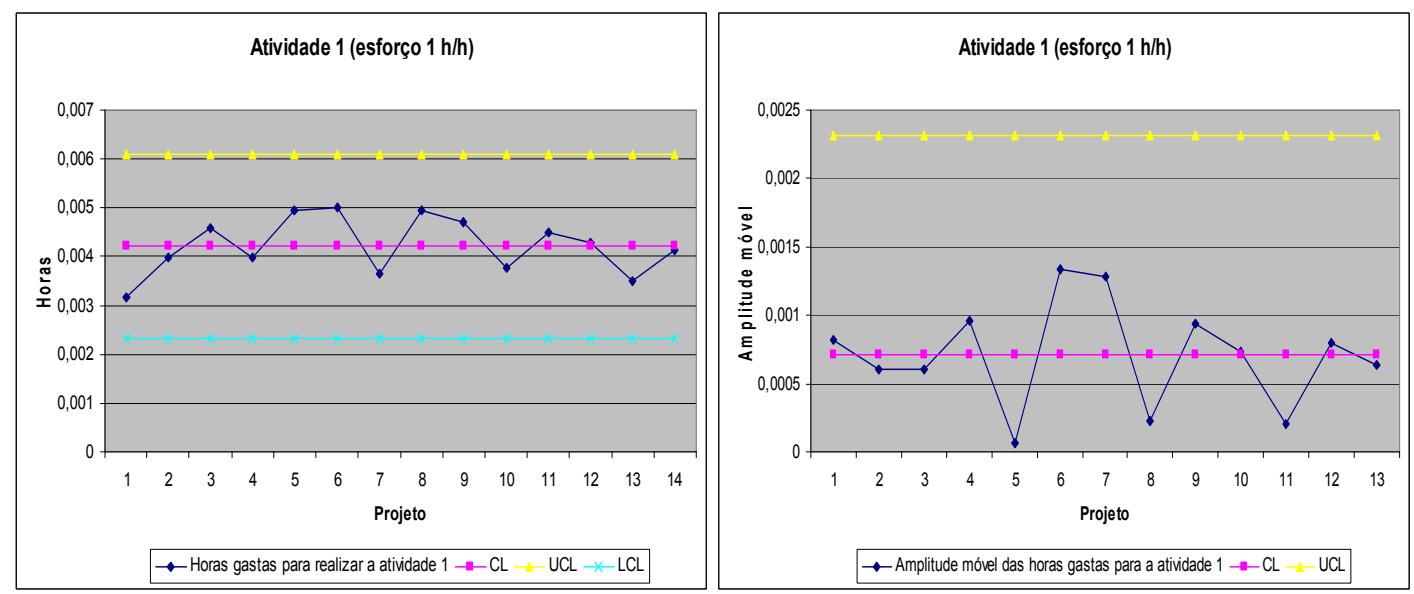

Figura 5 - Gráficos de controle da baseline de desempenho dos projetos para a atividade 1 do processo com os dados do projeto em seu $14^{\circ}$ ponto

Através da baseline de desempenho do processo, o gerente de projetos pode observar que seu projeto possui andamento conforme o previsto.

O exemplo acima demonstra a utilização da abordagem proposta quando o processo se encontra estável e uma baseline do desempenho do processo está disponível para estimativas e monitorações dos projetos. No segundo exemplo, descrito abaixo, o processo se encontra estável e foi monitorado e controlado apenas com os próprios dados:

"Suponha que o gerente de um projeto, na monitoração semanal, notou que ocorreu um atraso muito grande com relação as atividade que estavam planejadas para 
serem concluídas até aquela semana e as atividades realmente concluídas. Isso pode ser observado através da tabela 6 , onde para cada semana é exibido o número de atividades previstas a serem concluídas (Semanal Planed Value), o número de atividades realmente concluídas (Semanal Earned Value), seus valores acumulados, o índice de atraso (SSPI) e a porcentagem de atraso correspondente. O projeto tinha 22 semanas (cinco meses e meio) previstas e estava concluindo sua $12^{\mathrm{a}}$ semana (terceiro mês).

Tabela 6 - Dados do projeto para as doze primeiras semanas

\begin{tabular}{|l|l|l|l|l|l|l|}
\hline Semana & SPV & SEV & $\begin{array}{l}\text { SPV } \\
\text { acumulado }\end{array}$ & $\begin{array}{l}\text { SEV } \\
\text { acumulado }\end{array}$ & SSPI & $\begin{array}{l}\text { Porcentagem de } \\
\text { atraso }\end{array}$ \\
\hline 1 & 7 & 7 & 7 & 7 & 1 & 0 \\
\hline 2 & 8 & 8 & 15 & 15 & 1 & 0 \\
\hline 3 & 6 & 5 & 21 & 20 & 0,952380952 & 4,761904762 \\
\hline 4 & 7 & 7 & 28 & 27 & 0,964285714 & 3,571428571 \\
\hline 5 & 6 & 6 & 34 & 33 & 0,970588235 & 2,941176471 \\
\hline 6 & 6 & 6 & 40 & 39 & 0,975 & 2,5 \\
\hline 7 & 5 & 6 & 45 & 45 & 1 & 0 \\
\hline 8 & 7 & 6 & 52 & 51 & 0,980769231 & 1,923076923 \\
\hline 9 & 8 & 6 & 60 & 57 & 0,95 & 5 \\
\hline 10 & 7 & 7 & 67 & 64 & 0,955223881 & 4,47761194 \\
\hline 11 & 6 & 6 & 73 & 70 & 0,95890411 & 4,109589041 \\
\hline 12 & 7 & 1 & 80 & 71 & 0,8875 & 11,25 \\
\hline
\end{tabular}

Com base nesses dados, o gerente do projeto gerou um gráfico XmR com o SSPI do projeto para as 12 semanas já ocorridas do projeto, correspondente ao indicador estatístico 1.1 sugerido pela abordagem. Os gráficos de controle gerados podem ser observados na Figura 6.
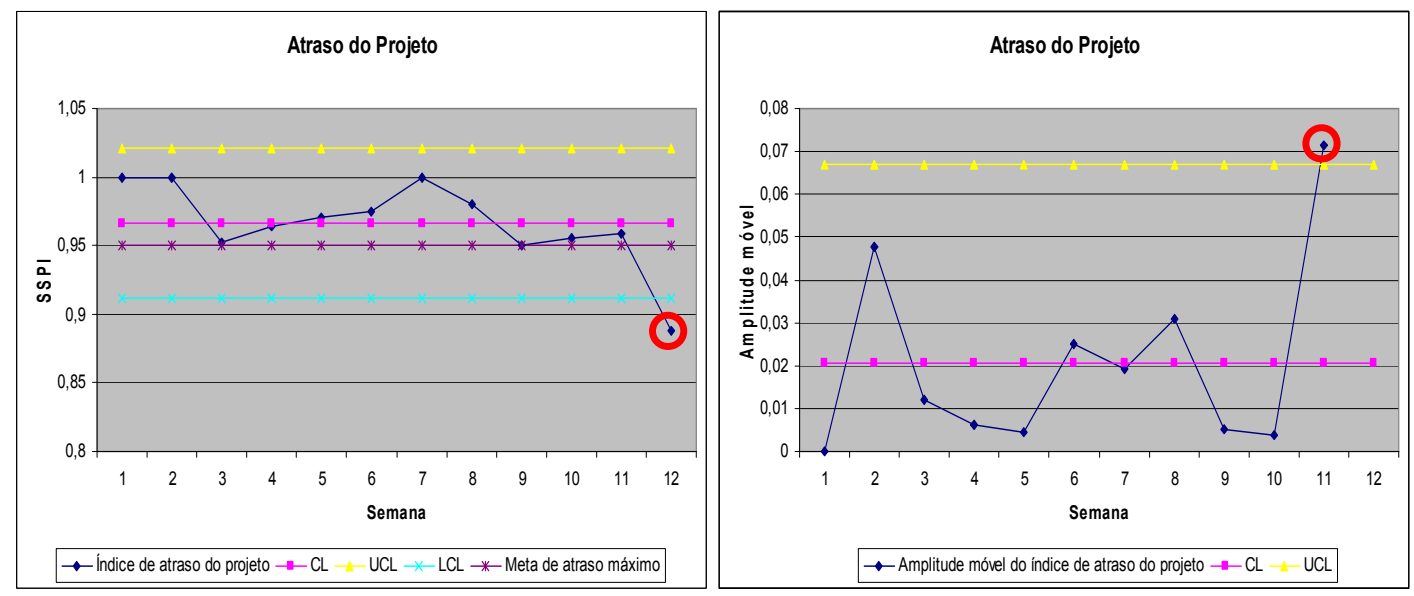

Figura 6 - Gráficos de controle para as doze primeiras semanas do projeto

Pode-se notar que na $12^{\mathrm{a}}$ semana, como observado pelo gerente de projeto, o atraso foi bem maior que o ocorrido nas semanas anteriores, ultrapassando a meta de atraso máximo de $5 \%$. Como existiam poucos pontos, dividi-los em gráficos de controle por fase teria pouca significância estatística. Mesmo com poucos pontos, porém, o fato de o ponto estar fora dos limites já indica um possível problema que pode ser estudado. O gerente de projeto resolveu buscar o motivo que ocasionou o ponto fora dos limites, pois podia se tratar de uma causa atribuível, e nesse caso, seria importante detectá-la e 
corrigi-la para evitar sua recorrência. A décima segunda semana do projeto se encontrava na fase 4 do processo do projeto, onde a análise e o projeto são desenvolvidos. O gerente solicitou informações com sua equipe que justificassem o atraso e foi informado que, ao realizar a análise e projeto do software, os analistas precisaram sanar algumas dúvidas com o cliente. O cliente, porém, alegava estar muito atarefado com outras atividades e por inúmeras vezes havia cancelado reuniões agendadas para este fim, atrasando o andamento do projeto mais que o normal. Para confirmar tais informações, o gerente de projeto solicitou a quantidade de reuniões canceladas pelo cliente, recebendo os dados da tabela 7 .

Tabela 7 - Dados do indicador não estatístico 6.1 para o projeto $X$

\begin{tabular}{|l|l|l|l|l|}
\hline & \multicolumn{4}{|l|}{ Reuniões com o Cliente } \\
\hline Fase & Marcadas & Efetuadas & Canceladas pela empresa & Canceladas pelo Cliente \\
\hline Primeira & 2 & 2 & 0 & 0 \\
\hline Segunda & 5 & 4 & 0 & 1 \\
\hline Terceira & 2 & 1 & 0 & 1 \\
\hline Quarta & 3 & 0 & 0 & 3 \\
\hline Total & 12 & 7 & 0 & 5 \\
\hline
\end{tabular}

Com a confirmação das informações recebidas pelo gerente de projeto da equipe do projeto, o gerente pôde realizar uma reunião com a alta diretoria, explicando o problema. Uma reunião com o cliente foi realizada onde o problema foi exposto e o cliente atentou pro fato que a presença dele era fundamental para o bom andamento do projeto.

O exemplo acima demonstra a utilização da abordagem proposta quando o processo se encontra estável e o processo é monitorado e controlado apenas com os dados do próprio projeto em andamento.

\section{Conclusão}

Este trabalho apresentou um exemplo de como controlar estatisticamente o processo e gerenciar quantitativamente os projetos. Os conceitos básicos do controle estatístico de processos e da gerência quantitativa dos projetos foram apresentados. Os trabalhos relacionados foram discutidos, a abordagem proposta foi apresentada e exemplos da execução da abordagem foram exibidos. Como parte da abordagem, foram apresentadas questões identificadas como essenciais para o controle estatístico do processo e para a gerência quantitativa dos projetos, juntamente com seus indicadores estatísticos e não estatísticos correspondentes.

A abordagem apresentada espera ajudar as organizações a iniciar o controle estatístico de processo e a gerência quantitativa de seus projetos e entender seus benefícios, auxiliando nas escolhas das informações a serem coletadas e na aplicação das técnicas estatísticas. A abordagem foi desenvolvida com base nas necessidades de uma organização de software localizada na cidade do Rio de Janeiro e com a participação de um membro da organização. A organização pretende executar a abordagem durante os próximos meses, em seus esforços para alcançar o nível 4 do CMMI [Chrissis et al. 2003]. 


\section{Referências}

Basili, V., Caldieira, G., Rombach, H., "The Goal Question Metric Approach", Encyclopedia of Software Engineering, pp. 528-532, John Wiley \& Sons, Inc., 1994.

CHAOS, The Standish Group International, Inc., Dennis, MA, 1994, 1997.

Cangussu, J. W., DeCarlo, R. A., Mathur, A. P., "Monitoring the Software Test Process Using Statistical Process Control: A Logarithmic Approach”, ESEC/FSE, 2003.

Chrissis, M.B., Konrad M., Shrum S., "CMMI ${ }^{\circledR}$ Guidelines for Process Integration and Product Improvement", Addison-Wesley, 2003.

Conradi, R., Fuggetta, A., "Improving Software Improvement," pp. 92-99, IEEE Software No.4, vol 19, 2002.

Deming, W. Edwards, "Out of the Crisis", MIT Center for Advanced Engineering Study, Cambridge, Massachusetts, 1986.

Florac, W. A., Carleton, A. D., "Measuring the Software Process," Addison-Wesley, 1999.

ISO/IEC 15504, "Information Technology - Software Process Assessment Part 1 Concepts and vocabulary; part 2 - Performing an assessment; part 3 - Guidance on performing an assessment; part 4 - Guidance on use for process improvement and process capability de-termination; and part 5 - An exemplar process assessment model."

ISO/IEC 9001: 2000, “Quality Management Systems - Requirements.

ISO/IEC 9004: 2000, “Quality Management Systems - Guidelines for Performance Improvements.

ISO/IEC 15939: 2002, "Software Measurement Process".

Komuro, M., "Experiences of Applying SPC Techniques to Software Development Processes", ICSE, 2006.

McGarry, J., Card, D., Jones, C., Layman, B., Clark, E., Dean, J., Hall, F., "Pratical Software Measurement", Addison-Wesley, 2002.

MPS.BR - Melhoria de Processo do Software Brasileiro, Guia Geral, versão 1.1, 2006.

Sargut, K. U., Demirors, O., "Utilization of statistical process control (SPC) in emergent software organizations: pitfalls and suggestions", Software Quality Journal, 2006.

Shewhart, W., "The Economic Control of Quality of Manufactured Product", D. Van Nostrand Company, New York, 1931, reimpresso por ASQC Quality Press, Milwaukee, Wisconsin, 1980.

Solingen, R., Berghout, E., "The Goal/Question/Metric Method: a practical guide for quality improvement of software development", McGraw-Hill Publishing Company, 1999.

Tayntor, C.B., "Six Sigma Software Development”. CRC Press LLC, New York. 2003.

Wang, Q., Jiang, N., Gou, L., Liu, X., Li, M., Wang, Y., "BSR: A Statistic-based Approach for Establishing and Refining Software Process Performance Baseline", ICSE, 2006. 